Document downloaded from:

http://hdl.handle.net/10251/63852

This paper must be cited as:

Gimenez Gandia, JJ.; Stare, E.; Bergsmark, S.; Gómez Barquero, D. (2015). Advanced Network Planning for Time Frequency Slicing (TFS) Toward Enhanced Efficiency of the Next-Generation Terrestrial Broadcast Networks. IEEE Transactions on Broadcasting. 61(2):309-322. doi:10.1109/TBC.2015.2402514.

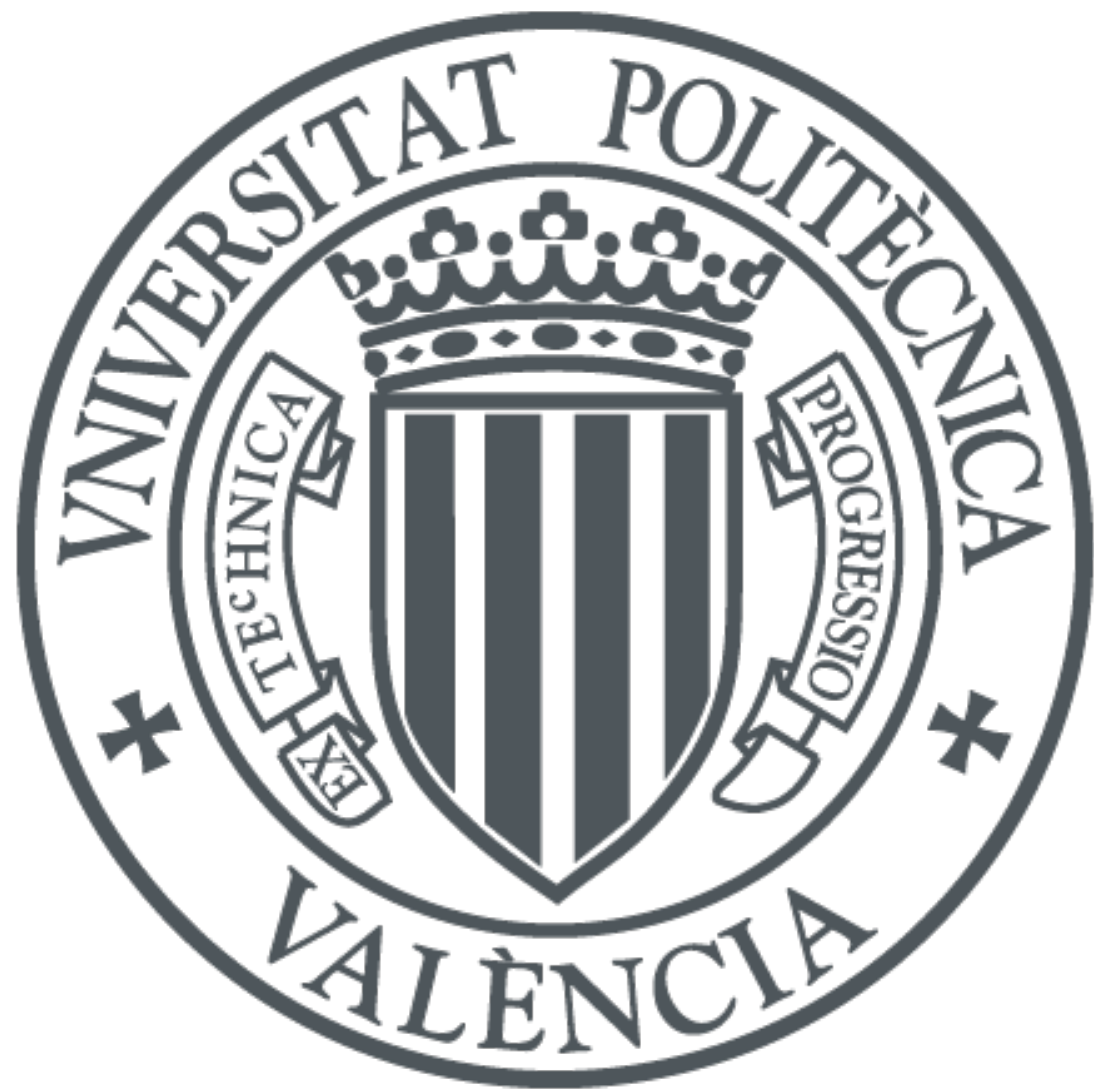

The final publication is available at

http://dx.doi.org/10.1109/TBC.2015.2402514

Copyright Institute of Electrical and Electronics Engineers (IEEE)

Additional Information

(C) (c) 2015 IEEE. Personal use of this material is permitted. Permission from IEEE must be obtained for all other uses, in any current or future media, including reprinting/republishing this material for advertising or promotional purposes, creating new collective works, for resale or redistribution to servers or lists, or reuse of any copyrighted component of this work in other works 


\title{
Advanced Network Planning for Time Frequency Slicing (TFS) towards Enhanced Efficiency of the Next-Generation Terrestrial Broadcast Networks
}

\author{
Jordi Joan Giménez, Erik Stare, Staffan Bergsmark and David Gómez-Barquero
}

\begin{abstract}
The allocation of frequencies traditionally used by terrestrial broadcasting (digital dividend) to International Mobile Telecommunication (IMT) is limiting the evolution of the Digital Terrestrial Television (DTT) networks for enhanced service offering. Next-generation DTT standards are called to provide increased capacity within the reduced spectrum. Time Frequency Slicing (TFS) has been proposed as one of the key technologies for the future DTT networks. Beyond a coverage gain due to additional frequency diversity, and a virtual capacity gain due to a more efficient statistical multiplexing, TFS also provides an increased interference immunity which may allow for a tighter frequency reuse enabling more $\mathrm{RF}$ channels per transmitter station, within a given spectrum. Moreover, the implementation of Advanced Network Planning (ANP) strategies together with next-generation DTT standards may result in additional spectral efficiency gains linked to network planning. This paper evaluates the potential spectral efficiency by TFS and ANP strategies in Multiple Frequency Networks (MFNs) as well as in regional and large area Single Frequency Networks (SFNs). Different network configurations have been analysed using single polarization, the systematic use of Horizontal and Vertical polarizations in different stations, or the use of multiple frequency reuse patterns for different frequencies of the TFS-Mux. Results indicate high potential network spectral efficiency gains compared to the existing network deployments with DVB-T2.
\end{abstract}

Index Terms-next-generation terrestrial broadcasting, tighter frequency reuse, TFS, advanced network planning, spectral efficiency, DVB-T2, DVB-NGH, ATSC 3.0

\section{INTRODUCTION}

D IGITAL terrestrial broadcasting spectrum has become the spotlight of spectrum regulation in the last years [1]. Important decisions are being taken on the allocation of parts of the UHF spectrum traditionally used for terrestrial broadcasting to satisfy the rapidly growing demand for wireless broadband International Mobile Telecommunications (IMT) [2].

The transition from analogue to digital TV implied itself an increase in spectral efficiency. Digital Terrestrial Television (DTT) provides e.g. improved co-channel interference (CCI) and adjacent channel interference (ACI) performance and enables the deployment of Single Frequency Networks (SFNs) [3]. TV services are packed in multiplexes and transmitted over single Radio Frequency (RF) channels. The

Jordi Joan Giménez and David Gómez-Barquero are with the iTEAM Research Institute of the Universitat Politecnica de Valencia (iTEAM-UPV), Valencia, Spain e-mail: jorgigan@iteam.upv.es

Erik Stare and Staffan Bergsmark are with Teracom AB, Stockholm, Sweden. first-generation DTT standard DVB-T (Digital Video Broadcasting Terrestrial), using MPEG-2 video coding, allowed the transmission of about 4 to 7 Standard Definition (SD) services per multiplex using about 20 to 24 Mbps for fixed roof-top reception conditions. The evolution of video coding standards also allowed launching HDTV (High Definition TV) services using MPEG-4/AVC (Advanced Video Coding), which provides more than $50 \%$ coding gain with respect to MPEG-2. The second generation DTT standard DVB-T2 (Terrestrial $2^{\text {nd }}$ Generation) went a step further outperforming DVB-T with a data rate increase of around 50\%-60\% for the same coverage, which allows the transmission of 4-5 HDTV services using about 36-40 Mbps [4].

This spectral efficiency increase offered by DTT made the International Telecommunications Union (ITU) reach a decision to release frequencies allocated to the broadcast service in the form of the so-called digital dividend [5]. This decision affected the $800 \mathrm{MHz}$ UHF band (790-862 MHz) in ITU Region 1, and the $700 \mathrm{MHz}$ band (698-806 MHz) in ITU Regions 2 and 3. The WRC-12 (World Radiocommunications Conference 2012) assigned the $700 \mathrm{MHz}$ band (694-790 MHz) in Region 1 to broadcasting and IMT on a co-primary basis, to take effect after WRC-15. This band is currently allocated on a co-primary basis in North America (Region 2) and Asia (Region 3) and allocated to IMT in North America and in some countries of Region 3. In Europe, some countries (e.g., Finland, Sweden and Switzerland) have already announced their intentions to assign the band to IMT and European Commission is also studying this point with a proposal to release the 700 band by 2020 and guarantee regulatory stability for broadcasting in the band 470-694 MHz until 2030.

Fig. 1 shows the available spectrum for DTT in ITU Region 1. The first digital dividend has already eliminated channels 61 to 69 and, if the $700 \mathrm{MHz}$ band is finally released, the remaining number of RF channels in the UHF band would be only 28 (470-694 MHz).

The new situation requires a spectral efficiency increase in the DTT networks by the migration to more efficient DTT standards together with the introduction of enhanced video coding standards. The transition from DVB-T to DVB-T2 already allows the retention of a similar DTT service offering in the remaining spectrum. However, the potential migration from SD to HDTV or even the start-up of Ultra HDTV services will require a stronger effort. During the last years, organizations such as the Future of Broadcast TV Initiative (FoBTV) [6], DVB or the ATSC (Advanced Television System Committee) 


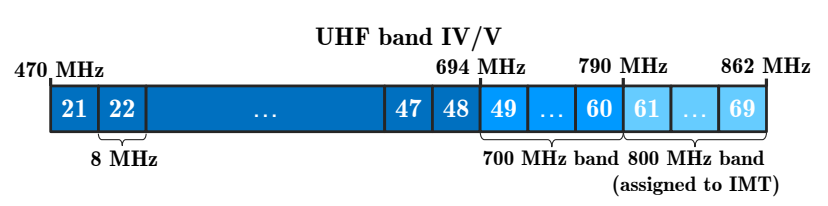

Fig. 1. Frequency allocations in the UHF band for digital terrestrial broadcasting in ITU Region 1. 49 channels are available. $40 \mathrm{RF}$ channels after completion of the $800 \mathrm{MHz}$ band digital dividend and $28 \mathrm{RF}$ channels after the $700 \mathrm{MHz}$ band digital dividend. The highest channel for DTT would be 48 what would leave a guard band of $9 \mathrm{MHz}$ to IMT.

are addressing the evolution of the DTT. The next-generation DTT standards, such as the upcoming ATSC 3.0, together with the introduction of the HEVC (High Efficiency Video Coding) video coding standard, which provides more than $50 \%$ coding gain with respect to H.264 [7], are envisage as the options for the next-generation DTT networks.

Traditional efforts on DTT standardization are mainly focused on maximising bit rate for a given $\mathrm{C} / \mathrm{N}$ (carrier-to-noise ratio) within the channel bandwidth. However, spectral efficiency in real networks is in practice limited by interference. DTT networks are traditionally deployed by means of high-tower/high-power transmitter stations which require frequency coordination between them. Thus, only a limited number of RF channels can be used per station. Frequencies are reused in distant stations to achieve an acceptable level of CCI.

International frequency plans for terrestrial broadcasting services are agreed in order to allow for efficient spectrum use. In ITU Region 1, the Geneva'06 plan (GE06) [8] allows, on average, 7 available RF channels per station in the UHF band out of the total 49 RF channels. On average, a group of 7 transmitters can use different frequencies. The reduced number of RF channels as a result of the release of frequencies from the $700 \mathrm{MHz}$ digital dividend, will require a tighter frequency reuse and an associated increased spectral efficiency to maintain the number of RF channels available per station.

New technologies such as Time Frequency Slicing (TFS) [9] can increase the network spectral efficiency (in terms of bps/Hz) by potentially tolerating a higher $\mathrm{C} / \mathrm{I}$ in the network deployments. TFS is part of an informative (not normative) annex of the DVB-T2 specification [10] and is fully adopted in the mobile broadcasting standard DVB-NGH (Next Generation Handheld) [11]. It is also proposed for adoption in the upcoming ATSC 3.0 standard.

TFS consists in the transmission of the data of a TV service across multiple RF channels instead of using a single one. With TFS each broadcast service is spread over a high capacity TFSMux by time-slicing and frequency-hopping. Whereas with a traditional Non-TFS transmission receiver tuner is locked to the RF channel containing the desired service, with TFS frequency hopping across the RF channels in the TFS-Mux is necessary to recover the data of the service. Transmissions using TFS benefit from a capacity gain due to an efficient statistical multiplexing for Variable Bit Rate (VBR) services and increased frequency diversity what is translated to a coverage gain for the reception of the complete set of services in the TFS-Mux. Increased frequency diversity also improves robustness against interferences since the received signal does not depend on a unique (potentially interfered) RF channel but on the whole set of RF channels involved in the TFS transmission, which may present different levels of C/I.

This paper focuses on the potential for a higher total capacity which may be achieved in a given spectrum thanks to the higher interference tolerance offered by TFS. This increased interference tolerance may be exploited as a combination of a tighter frequency reuse together with a modified capacity per RF channel. The overall effect is a higher total capacity within a given spectrum taking into account also the frequency reuse.

This effect is enhanced by the implementation of Advanced Network Planning (ANP) strategies by means of multiple frequency reuse patterns and/or the systematic use of $\mathrm{H} / \mathrm{V}$ polarizations. Spectral efficiency implications of the network configurations are studied for pure MFNs (Multiple Frequency Networks), for a network of SFN (Single Frequency Networks) clusters with up to seven transmitter stations per cluster and for large area SFN clusters with important limitations due to self-interference but with small interference from frequency reuse areas. It should be noted that also large area SFNs (e.g. country-wide) need frequency reuse (e.g. with other countries) to avoid harmful interference at the border between different SFN areas. Results are obtained for ideal networks made of hexagonal transmitter areas. Their application to real deployments are also discussed. Results are compared to existing DVB-T2 network deployments.

The paper is structured as follows. Section II discusses spectral efficiency offered by the conventional network planning of the DTT networks. Section III introduces advanced network planning configurations for increased spectral efficiency with or without TFS. Section IV describes the simulation environment for TFS/ANP evaluation and methodology. Section V presents the obtained results and discusses their applicability. Conclusions are finally presented in Section VI.

\section{Conventional Network Planning in DTT NETWORKS}

DTT stations deliver services by using a set of multiplexes (one per RF channel) in the broadcast bands. To extend the coverage to an arbitrarily large area, frequency reuse is implemented at different transmitter areas due to the limited amount of spectrum. The way the reuse is performed is mainly linked to the system robustness against CCI (expressed in terms of protection ratios). The required reuse limits the number of available RF channels per transmitter station.

Spectral efficiency is traditionally referred to the capacity per RF channel, without considering the influence of network planning. The maximum theoretical (Shannon [12]) rate in bits per second (bps) that can be transmitted to the reception point is given by:

$$
R=B \cdot \log _{2}(1+\gamma)
$$

where $\gamma$ is the carrier-to-interference plus noise ratio $C /(N+$ $I$ ), in linear units, and $B$ is the channel bandwidth (in $\mathrm{Hz}$ ).

The available $\mathrm{C} / \mathrm{I}$ and the frequency reuse factor limit the total capacity of the network for a given available spectrum. 


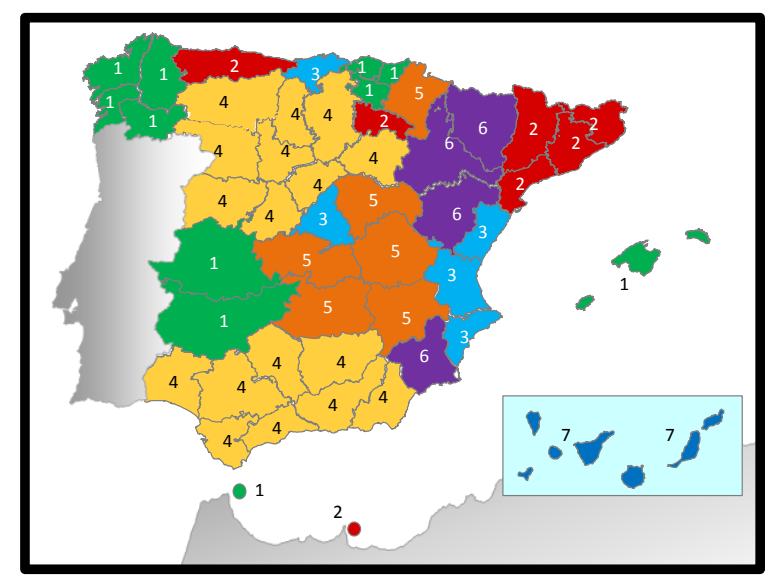

Fig. 2. Frequency reuse in Spain for the public state TV multiplex. Frequencies are reused in a basis of regional SFN clusters between different regions. $7 \mathrm{RF}$ channels in the UHF band are used at different transmitter stations.

The network spectral efficiency (NSE, in bps/Hz) accounts for the combined effect of capacity per multiplex (limited by C/I) and frequency reuse factor. The NSE is defined as:

$$
N S E=\frac{1}{\eta} \cdot \frac{R}{B}
$$

The frequency reuse factor, $\eta$, defines the average number of transmitter areas that use a different nominal frequency to transmit the same multiplex. With this approach, $\eta$ different RF channels are necessary to build a network of one multiplex that uses a single RF channel per multiplex and transmitter area. With $N_{R F}$ multiplexes per transmitter area a total of $\eta * N_{R F}$ frequencies are required to build the complete network [13]. Equation 3 defines the frequency reuse factor as:

$$
\eta=\frac{M}{N_{R F}}
$$

where $M$ denotes the number of available RF channels.

The GE06 UHF plan defines typically 7 network layers each using about 7 frequencies (multiplexes) in the UHF band. The resultant plan was based on different requirements, generally aiming for nationwide coverage in each country, with different conditions in terms of size and shape of envisaged coverage areas as well as the wave propagation conditions.

Fig. 2 shows a real example of the frequency reuse for the public state TV multiplex in different areas in Spain by means of regional SFN clusters to allow regional content insertion. 7 RF channels in the UHF band are reused along the country.

DTT networks are usually deployed by using linear polarization (e.g. horizontal or vertical) in all transmitter stations. Reception is typically achieved by directional roof-top antennas pointed towards the main transmitter station. The receiver antenna diagram weighs the interference contributions according to the angular (azimuth $\varphi$ ) direction between the main station and the co-channel stations. ITU-R BT.419 [14] recommends the values for antenna discrimination, $Q(\varphi, \kappa)$, relative to the direction of main response for broadcast reception to be used for frequency planning, as depicted in

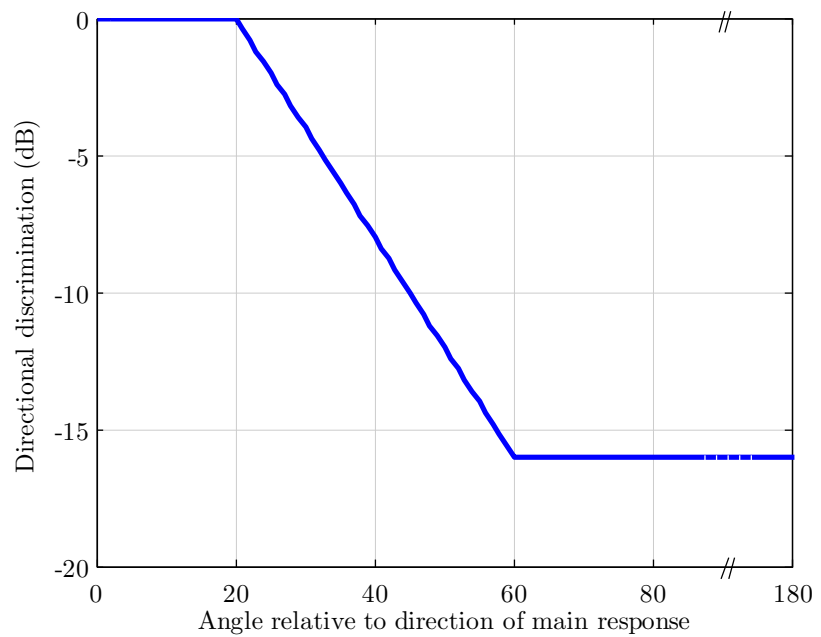

Fig. 3. Characteristics of directivity of the receiving antennas in UHF band according to ITU-R BT.419 [14]. Antenna discrimination relative to direction of main response, $Q(\varphi)$. Valid for signals of vertical or horizontal polarization.

Fig.3. With this antenna, cross-polar discrimination (XPD) is set to $Q(\forall \varphi, \kappa=-1)=-16 \mathrm{~dB}$, independently of direction ${ }^{1}$. Real antennas can differ from this model, as reflected in section IV-D.

In an interference-limited network, reception of the complete service offering (i.e. any service from any multiplex should be receivable) is limited by the most degraded RF channel. The use of TFS in future DTT networks could play an important role providing increased robustness against interferences and therefore a higher potential spectrum efficiency. Both the wanted signals and all interfering signals from a particular interfering site would be affected by independent frequency-dependent fading such that the resulting $C /(N+I)$ varies among the frequencies, even with nominally equal ERP (Effective Radiated Power) ${ }^{2}$. With TFS, the reception of a particular service is affected by the $\mathrm{C} /(\mathrm{N}+\mathrm{I})$ of different RF channels, due to interleaving. TFS capacity can be estimated as the average capacity over $N_{R F}$ RF channels which depends on the $\mathrm{C} /(\mathrm{N}+\mathrm{I})$ of each $\mathrm{RF}$ channel and the employed QAM mapping. According to [16], achievable TFS performance is limited by the saturation of capacity with a given QAM mapping. In addition, the combination of low FEC code rates and high order QAM modulation or the use of rotated constellations provides advantage against the high attenuation of RF channels [17]. As an example of this limitation, the capacity offered by DVB-T2 is bounded by the highest available QAM mapping (8 bps/Hz with 256QAM) and FEC code rate (5/6). Whereas, BICM capacity should be used, the analysis presented in this paper is intended for application to a future terrestrial broadcasting standard that could allow higher modulation orders and a shorter gap to

\footnotetext{
${ }^{1}$ Directional and polarization discrimination performance will be worse when using omni-directional antennas for portable indoor or mobile reception as identified in [15] where XPD is found to be in the range between $-7 \mathrm{~dB}$ and $-12 \mathrm{~dB}$.

${ }^{2}$ Received signal strength between RF channels mainly depends on transmitter antenna diagram, receiver antenna gain, signal propagation, etc.
} 


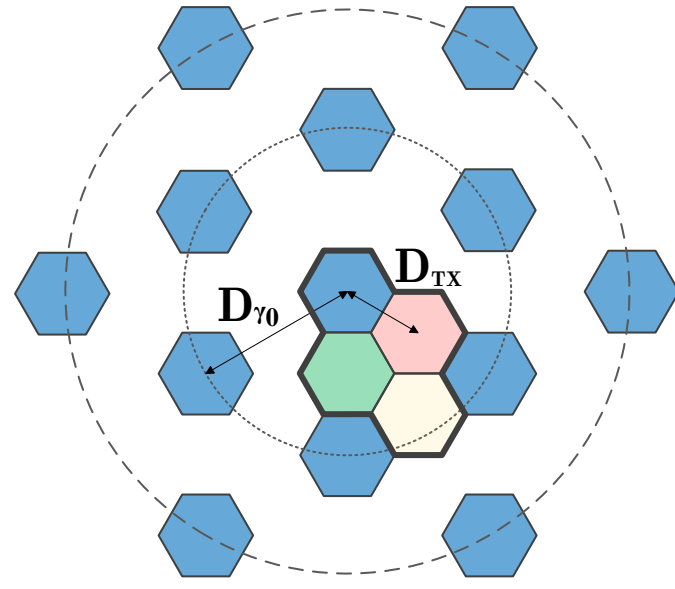

Fig. 4. MFN scheme with frequency reuse factor $\eta=4 . D_{T X}$ denotes the separation between transmitters (transmitter distance) and $D_{\gamma_{0}}$ is the minimum reuse distance. The two first co-channel tiers are depicted.

Shannon capacity. Shannon capacity is used instead.. Thus, NSE calculation with TFS can be simplified as defined in Equation 4:

$$
N S E_{T F S}=\frac{1}{\eta} \cdot \frac{1}{N_{R F}} \sum_{i=1}^{N_{R F}} \log _{2}\left(1+\gamma_{i}\right), \quad \text { bps } / H z
$$

$\gamma_{i}$ per RF channel directly depends on the network topology and frequency reuse pattern.

\section{A. Traditional Frequency Network planning without SFN}

Traditionally, different frequencies are assigned to multiplexes in each neighbour station in the target service area. At a given reception point, the wanted transmitter provides a certain field strength. Other transmitter stations using the same frequencies provide an interference contribution. Interferences are added so that the total interference power yields a $C /(N+I)$ at the reception point. It is necessary to maintain a sufficiently high $C /(N+I), \gamma_{0}$, to meet the interference protection requirements of the system. Thus, the RF channels from a transmitter station cannot be reused in a transmitter that is too close to the first one. This minimum separation between co-channel transmitters (i.e. minimum reuse distance $D\left(\gamma_{0}\right)$ ) limits the amount of spectrum that can be used per transmitter station. Fig.4 shows an scheme of a standard MFN made of regular adjacent hexagonal cells of radius $R$. Each cell has a single omni-directional antenna transmitter positioned in the cell centre.

Assuming that the RF channels are used in all the co-channel cells, the lower $C /(N+I)$ in the centre cell at a given location $j, \gamma_{M F N}$, is defined by:

$$
\gamma_{M F N}=\frac{P_{0} \cdot G_{0, j} \cdot Q\left(\varphi_{0, j}, \kappa\right)}{\sum_{i=1}^{N_{I}} P_{i} \cdot G_{i, j} \cdot Q\left(\varphi_{i, j}, \kappa\right)+P_{n}}
$$

where $N_{I}$ is the number of interfering stations, $P i$ is the power of each transmitter station $i, G_{i, j}$ is a propagation related constant from transmitter $i$ to receiver $j, Q\left(\varphi_{i, j}, \kappa\right)$ is the receiver antenna pattern attenuation relative to the pointing direction between a transmitter and the receiver, and $P_{n}$ is the noise power at the receiver. Polarization discrimination, $\kappa$ could also be considered when orthogonal polarization is used.

\section{B. Multiple Frequency Network planning using Single Fre- quency Network (SFN) clusters}

SFNs make use of the same frequency in a group of adjacent transmitters to cover a complete or part of a target service area. The service area could be a country, region or city. Thanks to the multipath capability of the OFDM signals by the Guard Interval (GI), signals arriving from co-channel transmitters contribute constructively to the total wanted signal if allowed delays are not exceeded. The maximum transmitter distances, without causing self-interference, allowed by DVB-T and DVB-T2 are $67.2 \mathrm{~km}$ (FFT 8k GI 1/4) and 159.6 km (FFT 32k GI 19/128), respectively, within an $8 \mathrm{MHz}$ RF channel. Corresponding maximum transmitter distances for a $6 \mathrm{MHz}$ channel are 8/6 larger. Given a transmitter distance, self-interference is reduced or eliminated by selecting a larger GI.

Multi-Frequency Network planning using SFN clusters is more spectral efficient than pure MFNs (i.e. cluster size $=1$ ) within a single network (e.g. a country). Conversely, the need of frequency reuse when there exist other adjacent networks (e.g. neighbour countries) makes NSE not as high as one might first believe from the term Single Frequency Network. In this study, SFN cluster size is limited in such a way that there is no self-interference within each cluster, assuming the largest DVB-T2 GI is used (32K with GI=19/128). The only interference comes therefore from the frequency reuse of the SFN clusters.

Large area SFNs may be large enough to experience negligible interference from frequency reuse (unless reuse equal to 1 or 2 is used). These networks suffer, instead, from self-interference (not enough GI), which may require a more dense transmitter infrastructure or larger overhead. Furthermore, SFNs limit the practical granularity for the delivery of regional or local services to the size of the SFNs themselves. The frequencies used in such medium-small SFNs may be reused across the network in a similar way as with an MFN. Fig.5 shows a medium-small regional SFN made up of a cluster of 7 transmitter stations and $\eta=4$.

The $C /(N+I)$ at a given receiver location within the SFN cluster is given by:

$$
\gamma_{S F N}=\frac{\sum_{i=1}^{N_{c}} P_{j, i} w_{i}(t)}{\sum_{i=1}^{N_{c}} P_{j, i}\left[1-w_{i}(t)\right]+\sum_{i=N_{c}+1}^{N_{I}-N_{c}} P_{j, i}+P_{n}}
$$

where $P_{j, i}=P_{i} G_{i, j} Q\left(\varphi_{i, j}, \kappa\right)$ is the received power that depends both on propagation and the antenna diagram, $N_{c}$ is the total number of transmitters in the SFN cluster and $N_{I}-N_{c}$ is the number of co-channel interferers from outside the SFN cluster. $w_{i}(t)$ is a weighting function which depends on the signal arrival time relative to the beginning of the FFT window; the equalization interval $\left(T_{E I}\right)$; the useful symbol 


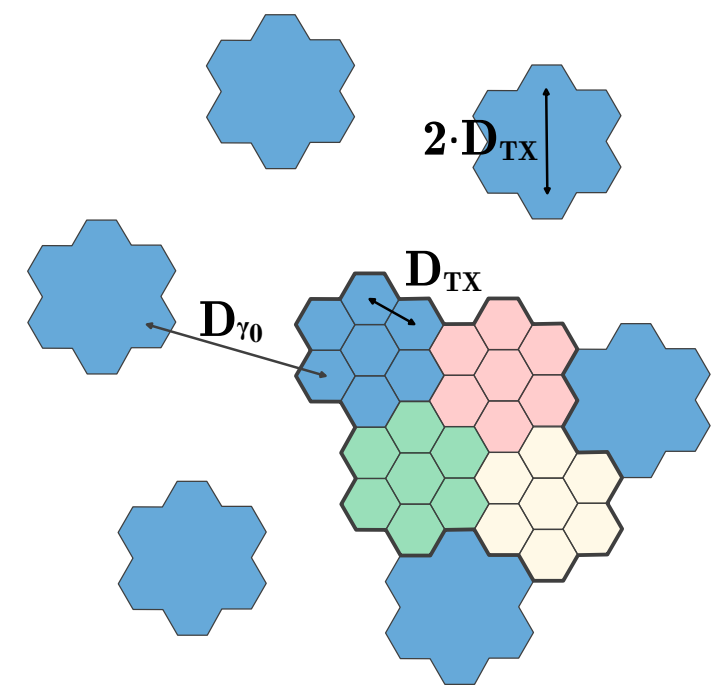

Fig. 5. SFN scheme with a transmitter cluster of 7 transmitter stations and frequency reuse factor $\eta=4$. Again, $D_{T X}$ denotes the separation between transmitters and $D_{\gamma_{0}}$ is the minimum reuse distance.

length $\left(T_{u}\right)$ and the guard interval length $\left(T_{g}\right)$ [18]:

$$
w_{i}(t)=\left\{\begin{array}{cl}
0 & t \notin T_{E I} \\
\left(\frac{T_{u}+t}{T_{u}}\right)^{2} & t \in T_{E I} \& t<0 \\
1 & t \in T_{E I} \& 0 \leq t \leq T_{g} \\
\left(\frac{\left(T_{u}+T_{g}\right)-t}{T_{u}}\right)^{2} & t \in T_{E I} \& t>T_{g}
\end{array}\right.
$$

The potential length of $T_{E I}$ depends on the RF bandwidth, FFT size and Pilot Pattern (PP) of the DVB-T2 signal, as well as on implementation constraints. In this study an EI having the same length as the $32 \mathrm{~K} 19 / 128$ GI in DVB-T2 has been used. Its position depends on the estimated impulse response. Different strategies of FFT window synchronization can be implemented at receivers as explained in [19]. The SFN calculations are performed considering the maximum $\mathrm{C} / \mathrm{I}$ approach so that the situation of the FFT window allows obtaining the maximum $\mathrm{C} / \mathrm{I}$ at the receiver.

\section{Advanced DTt Network Planning Strategies}

The introduction of next-generation DTT standards with improved CCI performance together with ANP strategies may allow for increased NSE. Receiving antenna directivity and polarization discrimination can be exploited to achieve effective reduction of the received interference and to maximize the $C /(N+I)$, particularly when combined with TFS. The use of TFS also allows for implementing a different frequency reuse pattern for each or some of the frequencies in the TFS-Mux.

1) Mixed Polarization Network (MPN): MPN consist of a systematic use of different polarizations, horizontal $(\mathrm{H})$ or vertical $(\mathrm{V})$, for transmitters using the same frequency. Users within the coverage area of a given transmitter receive all frequencies with the same polarization. Co-channel interferences coming with the opposite polarization from a distant station are discriminated by the antenna diagram $Q(\varphi, \kappa)$. In general, it would be desirable to have as many as possible of

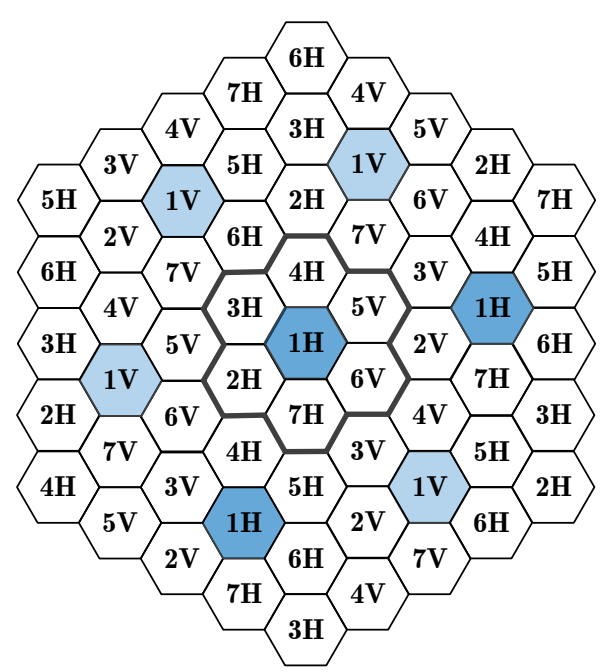

Fig. 6. MPN configuration using systematic polarization variation in the stations in the network. ' $H$ ' denotes horizontal and ' $\mathrm{V}$ ' vertical polarization. With the proposed MPN scheme for $\eta=7$, only 2 over 6 co-channel stations produce co-polar interference (dark cells).

the strongest interferers using the opposite polarization. Thus, the MPN scheme should be selected taking the frequency reuse pattern into account to minimize the number of neighbouring co-polar interferers which should ideally be the same for each station in the network. Although many possibilities exist, the MPN scheme depicted in Fig. 6 has been found to provide a good performance for the frequency reuse pattern $\eta=4$ and $\eta=7$ reported about in this paper. For frequency reuse $\eta=3$ it is possible to design a pattern with a column-by-column cross-polarization configuration (instead of using 2 adjacent rows as shown in the figure).

Thanks to the antenna polarization discrimination a given receiver in the centre cell benefits from an interference reduction, which increases $C /(N+I)$. Both a traditional and a network implementing TFS can benefit from the reduction of the interference level at receiving points.

Note that MPNs are not widely used in the existing DTT network. However, orthogonal polarization is used to achieve required protection in particular areas. DTT systems usually inherit antenna deployment from analogue TV, which, in general, used horizontal polarization to prevent ghost pictures from vertical polarization, particularly in VHF band. The use of orthogonal polarization is usually considered in on-channel repeaters in countries such as Spain and Denmark.

A similar MPN scheme can be synthesized for a network using SFN clusters to minimize the number of co-channel interferer clusters.

2) Multiple Frequency Reuse Patterns (MFRP): The use of MFRP with TFS exploits the $C /(N+I)$ differences among RF channels due to interferences coming from different directions. MFRP consists in the application of a different reuse pattern (with the same $\eta$ ) to the different frequencies (or groups of frequencies) of a TFS-Mux at a particular site. Each transmitter broadcasts the same number $N_{R F}$ of RF channels, but for a given area the different frequencies have different 


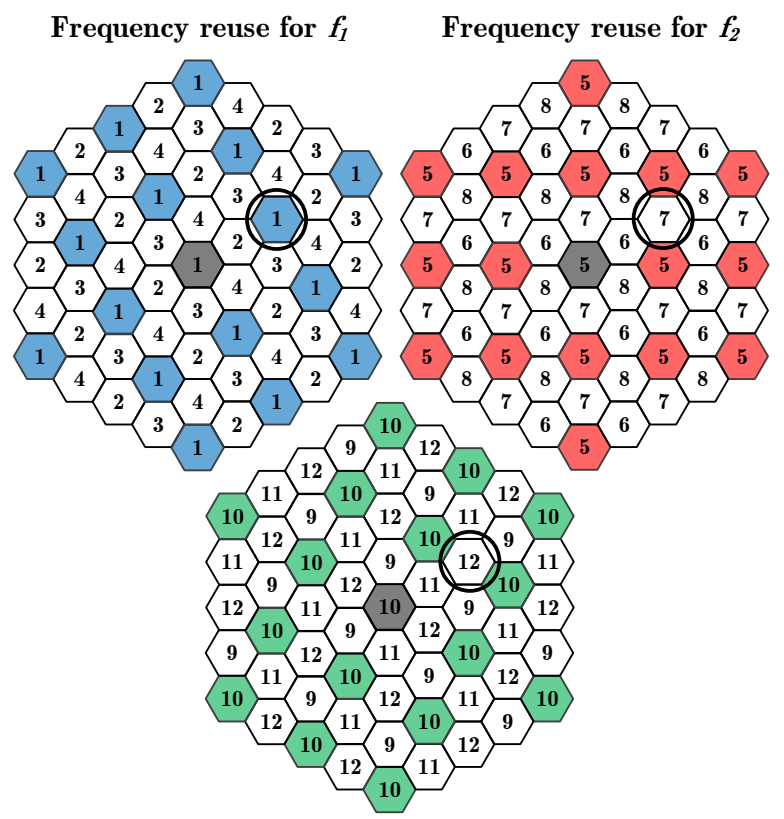

Frequency reuse for $f_{3}$
Frequency reuse for $f_{1} \quad$ Frequency reuse for $f_{2}$

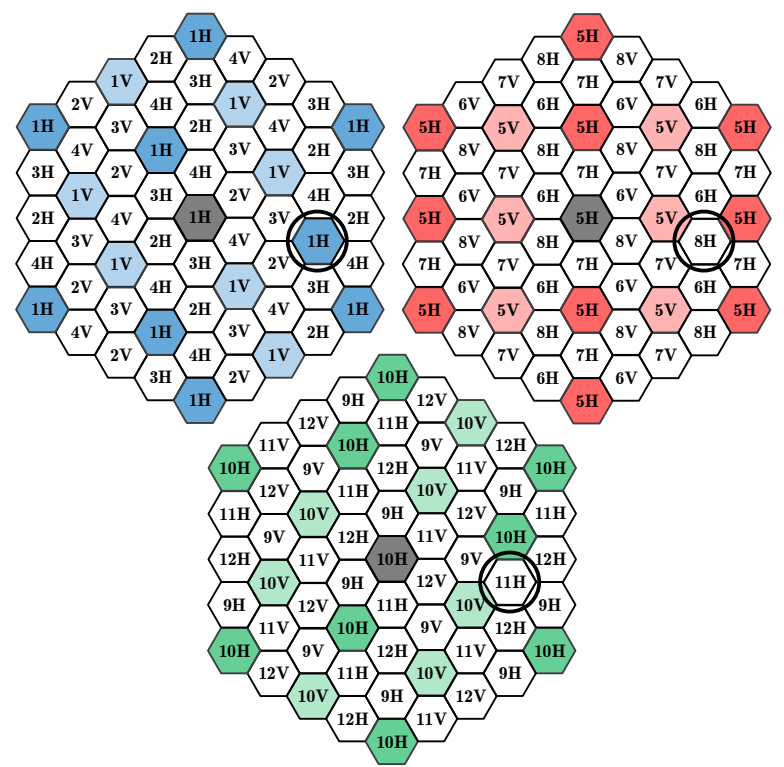

Frequency reuse for $f_{3}$
Fig. 7. MFRP network configuration for 3 frequency reuse patterns with $\eta=4$. Note that the wanted transmitter is located in the centre hexagon. Each frequency $\left(f_{1}, f_{2}\right.$ and $\left.f_{3}\right)$ in the centre hexagon receives interferences from a different cell.

frequency reuse patterns. The received interference level of the different TFS-Mux frequencies typically varies significantly as a result of differences in the location of the corresponding co-channel stations and due to differences in antenna diagram weighting.

Depending on $\eta$ the number of reuse patterns that can be applied to each ensemble of frequencies is different. With $\eta=$ 7 , two patterns are found whereas with $\eta=3$ there is only one pattern. For $\eta=4$, three different patterns can be found as shown in Fig.7. Note that these patterns are just mirrors $(\eta=7)$ or rotations $(\eta=4)$ of each other ${ }^{3}$.

For $N_{R F}=3$ this means that each TFS frequency gets a unique frequency reuse pattern. For $N_{R F}=6$, each pattern is used by two TFS frequencies $\left(N_{R F}=9\right.$ : three frequencies, etc). Thanks to the varying frequency reuse patterns, interference contributions from a particular neighbouring transmitter only affects (for $\eta=4$ ) one third of the $N_{R F}$ TFS frequencies.

3) Multiple Frequency Reuse Patterns and Mixed Polarization Networks: Both of the described methods (MFRP and MPN) may be combined in the same network. The combination is straightforward: the polarizations in Fig.6 are overlaid on top of the frequency reuse patterns of Fig.7. The three resulting frequency reuse patterns with different polarization for $\eta=4$ are shown in Fig.8. It should be noted that on average only one third of the eight closest interfering transmitters use the same polarization as the wanted transmitter. Fig.9 (top) shows the resulting distribution of co-

\footnotetext{
${ }^{3}$ There exists a fourth symmetric pattern, which is ideal for Non-TFS and may also be used for TFS, but with less gain than the three described non-symmetrical patterns. Any rotation of a symmetric pattern with $\eta=3$ and $\eta=4$ results in the same pattern.
}

Fig. 8. Network scheme with a combinations of the MPN and MFRP. Each frequency $\left(f_{1}, f_{2}\right.$ and $\left.f_{3}\right)$ in the centre hexagon receives interferences from a different cell and in some cases with orthogonal polarization.

polar and cross-polar co-channel transmitters with respect to the central cell with $\eta=4$.

With MFRP, interferers are not co-located for the used frequencies, i.e. a given co-channel transmitter station only interferes on one third of the TFS-Mux frequencies, and with MFRP+MPN the effect of polarization discrimination is added, thus, leading to a less number of high signal strength interferers.

The same principles, discussed here for pure MFNs, are also applicable to networks using SFN clusters. This allows further NSE increase for this type of networks, where each cluster takes a similar role as an individual transmitter in the pure MFN case, i.e. polarizations and frequency reuse patterns are alternating on the basis of SFN clusters instead of individual transmitters. Fig.9 (bottom) depicts the SFN clusters scheme of the MFRP+MPN strategy.

\section{Simulation and Methodology Considerations}

\section{A. Network configuration}

NSE evaluation for the different network configurations is conducted by computer simulations over an ideal hexagonal network. The assumed transmitter antenna diagrams are omnidirectional. Transmitter distance (inter-site distance) is selected to be $60 \mathrm{~km}$ and $80 \mathrm{~km}$ and the corresponding effective antenna height are $250 \mathrm{~m}$ and $400 \mathrm{~m}$, since they resemble typical Western and North European scenarios [20]. Received $C /(N+I)$ is calculated using 6 evenly distributed frequencies in the UHF band 470 to $694 \mathrm{MHz}$. This frequency allocation exploits a high TFS coverage gain and allows enough amount of frequencies to apply the different MFRPs obtained.

Fixed roof-top reception is assumed at $10 \mathrm{~m}$ above ground level with a directional antenna which characteristics are 

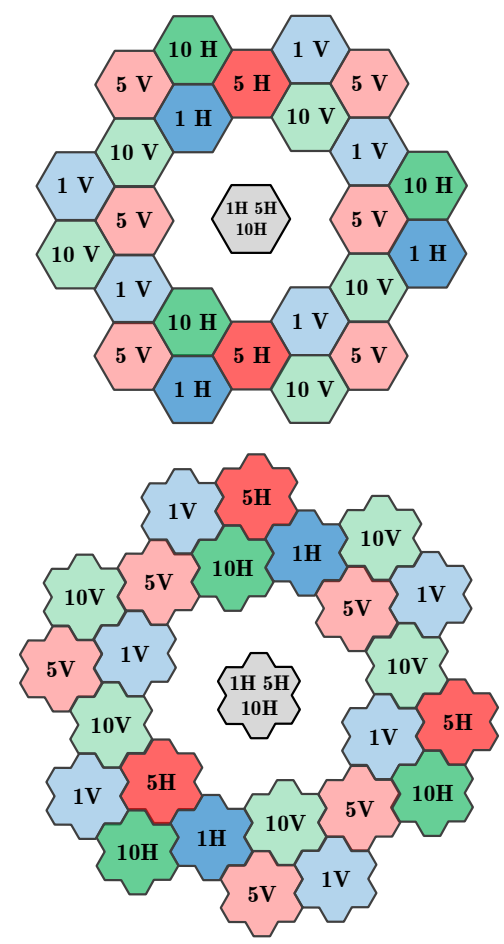

Fig. 9. MFN (top) and SFN (bottom) schemes of the stations interfering the wanted transmitter area for different frequencies with MPN and MFRP. The number of co-polar co-channel stations is reduced with this configuration. The number and position of the cross-polar interfering stations depends on the selected frequency reuse pattern.

described in ITU-R BT.419 [14], as shown in Fig.3. Other important parameters for received field strength calculation are also described in Table I.

\section{B. Propagation and shadow fading models}

The land propagation model is the ITU Recommendation ITU-R P.1546 [21] which defines the received electrical field strength at a certain distance given ERP, effective antenna height, frequency, receiver antenna height, terrain type and percentage of time.

The local received power from transmitter $i$ is given by the deterministic field strength from [21] and additional terms for frequency-independent but directional-dependent shadow fading and for frequency-dependent fading (independent of transmitter).

Shadow fading is modelled as an independent log-normal random variable (i.e. Gaussian distribution in $\mathrm{dB}$ with standard deviation $5.5 \mathrm{~dB}$ and $0 \mathrm{~dB}$ mean) for each transmitter station [21] defined as $\left\{F_{0}, F_{1} \ldots F_{N_{I}}\right\}$ with $N_{I}$ being the number of CCI stations. The shadowing components of the signals from different transmitters are assumed to be correlated for similar propagation paths (since they are affected by the same obstacles). A site-to-site cross correlation coefficient $\left(\rho_{c}\right)$ is modelled according to the angle-of-arrival difference $(\phi \in[0, \pi])$ and the ratio between the propagated path lengths $\left(\frac{D_{1}}{D_{2}}\right)$ by equation 8 [22]. The angle-of-arrival is assumed to be equal to the transmitter direction (i.e. multipath effects are
TABLE I

SIMULATION CONFIGURATION AND NETWORK PARAMETERS

\begin{tabular}{lc}
\hline \hline Propagation and coverage features & \\
\hline Frequencies & $474514554594634674 \mathrm{MHz}$ \\
\hline Reception Type & Fixed rooftop at $10 \mathrm{~m}$ \\
\hline Propagation Model & ITU-R P.1546-4 (Land) \\
\hline Propagation Standard Deviation & $5.5 \mathrm{~dB}$ \\
\hline Location Probability & $95 \%$ \\
\hline Time availability & $99 \%$ \\
\hline Transmitter characteristics & \\
\hline Tx antenna & Omni-directional \\
\hline Effective Tx antenna height & $250 / 400 \mathrm{~m}$ \\
\hline Tx distance & $60 / 80 \mathrm{~km}$ \\
\hline Receiver characteristics & \\
\hline Rx antenna direction in MFN & Strongest transmitter \\
\hline Rx antenna direction in SFN cluster & Highest C/I \\
\hline FFT time window synch. strategy $[19]$ & Maximum C/I \\
\hline Rx antenna model & ITU-R BT.419-3 \\
\hline Rx antenna gain & $11 \mathrm{~dB} d$ \\
\hline Eff. antenna aperture & $\frac{\lambda^{2} G}{4 \pi}$ \\
\hline Feeder loss & $4 \mathrm{~dB}$ \\
\hline Equivalent noise BW & $7.61 \mathrm{MHz}$ \\
\hline Rx Noise Figure & $6 \mathrm{~dB}$ \\
\hline
\end{tabular}

not taken into account).

$$
\rho_{c}=\left\{\begin{array}{ccc}
\sqrt{\frac{D_{1}}{D_{2}}} & \text { for } & 0 \leq \phi \leq \phi_{T} \\
\left(\frac{\phi_{T}}{\phi}\right)^{\zeta} \cdot \sqrt{\frac{D_{1}}{D_{2}}} & \text { for } & \phi_{T} \leq \phi \leq \pi
\end{array}\right.
$$

$D_{1}$ represent the distance to the closest transmitter. $\phi_{T}$ is an angle threshold defined as $\phi_{T}=2 \sin ^{-1} \frac{r_{c}}{2 D_{1}}$, where $r_{c}$ represents the serial shadowing correlation distance. The exponent $\zeta$ accounts for the height and shapes of the terrain and buildings. The parameters are set to $\zeta=0.3$ and $r_{c}=300$ $\mathrm{m}$ [22].

Shadow fading is assumed to be frequency independent. Thus all TFS frequencies originating from a particular site are assumed to have the same shadow fading realisation.

Shadow fading for the different transmitter stations is calculated as:

$$
Y_{i}=\sqrt{\rho_{c}} \cdot F_{0}+\sqrt{1-\rho_{c}} \cdot F_{i}
$$

where $F_{0}$ represents the receiver-position-dependent fading component and $F_{i}$ models the station-dependent component [23].

Real transmitting antennas introduce a sort of frequency dependent fading, since the real antenna diagram typically varies significantly with frequency and direction. Also the effects of the wave propagation (multipath) and the positioning of the antenna are frequency dependent. All these effects are modelled as a frequency-dependent fading with $2 \mathrm{~dB}$ standard deviation. This supposes a conservative approach according to the results presented in [24] on RF channel imbalances.

\section{Network spectral efficiency calculation}

The following system and network combinations are studied and compared: 
- Reference case:

- Non-TFS: traditional network in which the RF channel with the worst RF level limits coverage.

- Combinations of ANP strategies which are compared to the reference case:

- Non-TFS+MPN: Non-TFS case with systematic polarization $(\mathrm{H} / \mathrm{V})$ repetition.

- TFS: spectral efficiency is given by the average spectral efficiency of the TFS RF channels.

- TFS+MFRP: MFRP configuration with the application of TFS.

- TFS+MPN: MPN configuration with the application of TFS.

- TFS+MFRP+MPN: Mixed MFRP and MPN with TFS.

Performance is, in general, evaluated over interference-limited networks, with a fixed high ERP so that noise power is negligible. V-B also discusses performance considering a range of ERP values.

Each configuration is compared for pure MFN, a network of SFN clusters and a large area SFN, in all cases with different frequency reuse factor. Pure MFN networks are studied for frequency reuse $\eta=3,4$ and 7 . Clusters of 7 transmitter stations are considered in MFNs made of SFN clusters for $\eta=4$ and 7. Large area SFN networks are analyzed for $\eta=3$ and 4. Results are also presented for frequency reuse factor 1 for comparison. Special attention is paid to $\eta=4$ and $\eta=7$ since they allow the implementation of MFRP.

For the given network and frequency reuse pattern, co-channel stations positions are calculated. The point inside the centre hexagon (for MFN) or centre cluster (for a network of SFN clusters) with the lowest capacity (lowest $C /(N+I)$ ) is taken as the worst location for Non-TFS. For TFS, the one with the lowest capacity taking into account the $C /(N+I)$ of all TFS frequencies is taken.

Note that the receiver antenna is pointed towards the centre of the cell in the case of a pure MFN and towards the transmitter station which provides the highest $C /(N+I)$ within an SFN cluster.

$C /(N+I)$ is computed assuming the field strength for the $50 \%$ of time for the wanted signal [21]. The path losses from all transmitters with significant interference contributions are calculated. The three first co-channel transmitter rings are taken into account for cluster size equal 1 (pure MFN case). For cluster size 7 , the first tier of clusters is considered. For the large area SFN calculation the number of interfering cells is computed according to the weighting function given by Equation 7. In all cases, adding more co-channel transmitters do not cause significant changes in the NSE calculation.

A realistic model for time correlation is unfortunately not well-established. Three particular cases are studied: a maximum-conservative case with $100 \%$ correlation and in the other end two variants of uncorrelated cases. For the fully-correlated case $(\mathrm{C})$ received field strength for all stations and frequencies is assumed to reach their $1 \%$ time value at the same time, i.e. with $100 \%$ correlation. In this case, the total interference, for this percentage of time, is the sum of the individual interferences' peak values (worst-case). For the uncorrelated case U1, a random statistical distribution ( $T=10000$ values) of field strength values from the curves in [21] is calculated by fitting Gaussian distributions in the range $1 \%$ to $10 \%$ and $10 \%$ to $50 \%$ percentage of time. With this, individual transmitters reach their different field strength values at independent points in time, i.e. their sum is typically much lower than in the worst case scenario. In this case, the frequencies transmitted in the same station are considered to be fully-correlated. With the uncorrelated case U2, all signals are assumed to be uncorrelated regardless their frequency and location. Although real signals are neither fully-correlated nor uncorrelated, the extreme cases are studied as a means to investigate the boundaries of the spectral efficiencies under analysis. ITU-R P.1406 Recommendation states that the correlation in mean received field strength from different stations mainly depends on the position of the sources. Signals coming from opposite directions are mainly uncorrelated whereas a high degree of correlation exists for co-sited sources [25].

At the top of each field strength realization, as described above, log-normal fading distribution with $K=100000$ realizations is applied to the wanted and interfering signals.

NSE (bps/Hz) is calculated by Equation 2. For the case of Non-TFS (i.e. a traditional DTT network), the NSE is limited by the worst RF channel, which constrains the coverage of the complete service offering. In the TFS cases, the NSE is obtained as the average of all the $N_{R F}$ TFS frequencies according to Equation 4. The NSE considered to be available at the reception point is the one available with $95 \%$ coverage probability for $99 \%$ of time.

Payload capacity overheads due to GI+PP (Guard Interval and Pilot Pattern) in MFN and SFN configurations are considered since they reduce payload capacity values. A typical MFN network configured with DVB-T2 32k GI 1/128 PP7 presents a GI+PP payload capacity overhead of $2.04 \%$. An SFN cluster with 7 transmitters and transmitter distance 60 $\mathrm{km}$ can be configured with the same mode but GI 1/8 PP2, in both $6 \mathrm{MHz}$ and $8 \mathrm{MHz}$ channel bandwidth, to withstand maximum SFN delay. This GI+PP configuration leads to $19.67 \%$ payload capacity overhead. With this GI configuration, those co-channel transmitters situated outside a cluster are taken as pure interfering stations. For the case of a large area SFN, the selected GI is 19/128 PP2, the maximum permitted by DVB-T2, which leads to an overhead of $21.49 \%$. According to [19], in this study FFT time window is assumed to be synchronized to the strongest received signal within the SFN since the antenna is positioned towards the main contributing station.

\section{Other considerations and limitations of the study}

The use of a the described simulation environment has implications on the accuracy of the obtained results and their applicability to real network deployments. In particular, the hexagonal model does not directly reflect a real deployment. The results are focused on a high power high tower (HPHT) deployment of transmitters. For these, the typical configuration 
is to assume equal ERP per station. However, the geographical distribution of transmitters in a real network does not follow a regular pattern due to the irregularity of the terrain, what leads to unequal transmitter distance.

Regarding SFNs, they are usually built-up by means of different types of transmitters, where low power transmitters (e.g. gap-fillers) are used to extend the coverage provided by the high power stations. Although SFN gain has been accounted for as constructive, according to [26] there are situations in which SFN signals can contribute destructively. This mainly occurs when contributions are received with equal strength and similar delays, what causes a $\mathrm{C} / \mathrm{N}$ loss. However, an SFN cluster with 7 transmitters and the use of directional antennas generate a channel in which a strong transmitter dominates and the other transmitters contribute in a limited way to the $\mathrm{C} / \mathrm{I}$.

The received signal level from interferers depends on propagation characteristics. The ITU-R P.1546 model is found to deviate for rural area calculations at large distances [27]. According to the model, the field strength values predicted are not specific for a given polarization. Thus, vertical polarization wave propagation is configured to be the same as horizontal polarization wave propagation. The existing of differences between both polarizations are not taken into account in this study.

In addition, multipath propagation has not been taken into account since its effect with fixed roof-top reception is limited. Additional degradation due to frequency selectivity and depolarization should be taken into account for a more accurate analysis. However, similar effects are expected for Non-TFS and TFS so that net effects on gains are likely to be small.

From the point of view of the receiver, receiver antenna diagram is paramount in connection with MFRP (azimuth discrimination) and MPN (polarization discrimination). In [28] real receiver antenna gains are shown to present different $\mathrm{H}$ and $\mathrm{V}$ radiation diagrams as function of azimuth and frequency. In fact, for some real antennas ITU-R BT.419 is pessimistic regarding azimuth discrimination since it is possible to obtain more than $16 \mathrm{~dB}$ antenna discrimination for about 50\% azimuth. Reference [29] concludes that some existing antennas present broader patterns and lower gain, particularly at low frequencies.

\section{Performance Evaluation}

\section{A. Network spectral efficiency study with interference-limited networks}

1) Pure Multiple Frequency Networks: Table II shows the NSE $(\mathrm{bps} / \mathrm{Hz})$ reached with different combinations of ANP strategies within pure interference-limited MFNs. Results are presented for frequency reuse factors 3,4 and 7 within the $D_{T X}=60 \mathrm{~km}$ and $h_{e f f}=250 \mathrm{~m}$ scenario. Fully-correlated (C) and uncorrelated (U1 and U2) approaches for the co-channel interference time correlation at the receiver point are considered. Note that with frequency reuse factor $\eta=3$ it is not possible to implement more than one frequency reuse pattern. Thus, the entries of the table involving this situation are not filled.

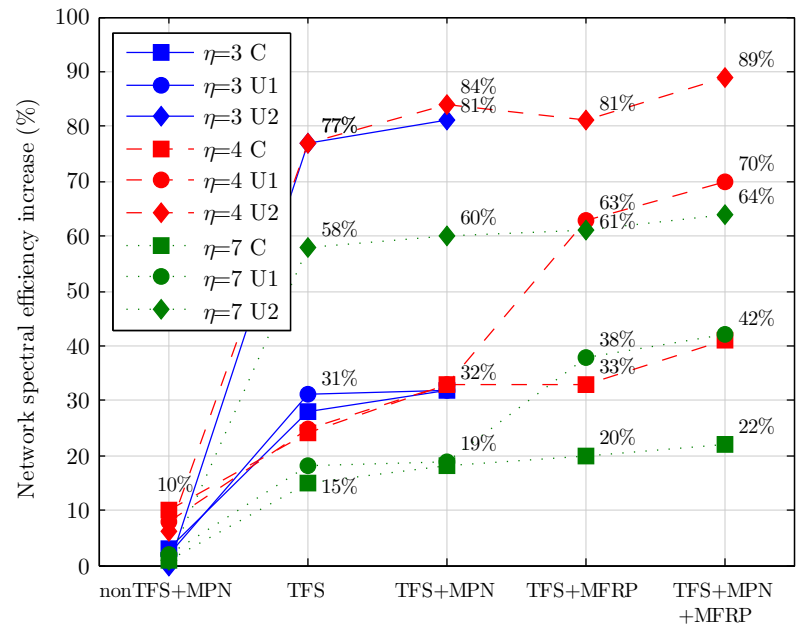

Fig. 10. NSE increase $(\% \mathrm{bps} / \mathrm{Hz})$ for different MFNs with reuse factor 3,4 and 7. For each reuse factor, the Non-TFS case is taken as reference. Transmitter distance $D_{T X}=60 \mathrm{~km}$ and effective antenna height $h_{\text {eff }}=$ $250 \mathrm{~m}$. Fully correlation (C) and uncorrelated variants U1 and U2.

The results show that, for all cases, the networks present higher NSE when decreasing the reuse factor from 7 to 4 and 3. Although the $C / I$ increases with the reuse factor, less spectrum can be used per station. These two effects roughly balance each other for each use case, but with a clear advantage for lower reuse factors. In particular for $\eta=7$, although achieving a high CCI performance, only $1 / 7$ of the spectrum can be used per station. Regarding CCI time correlation, the uncorrelated approach provides the highest NSE values. The values for Non-TFS configuration, which presents the lowest performance, are taken as reference. Fig.10 depicts the NSE increase for the different network configurations with respect to the reference case for each frequency reuse factor (i.e. each value corresponds to the increase with respect to the Non-TFS case).

The NSE gain achieved by MPN is larger for $\eta=4$ (around $8 \%-10 \%$ ), since the effect of the orthogonal polarization discrimination at the receiver provides a high $C / I$ increase. The number of co-polar transmitter stations in the first ring with $\eta=4$ and $\eta=3$ is 2 out of 6 . However, with $\eta=3$ these transmitters are closer. Similar gains are achieved with the three correlation approaches.

The use of TFS achieves higher values than the cases without TFS. The best performance is reached for the fully uncorrelated case with $\eta=3$ and $\eta=4$ ( $77 \%$ increase). For the correlated case (C), the highest increase is reached for $\eta=4$ (31\%). The main advantage of using TFS-only is that the increased NSE comes without network planning/implementation modifications.

Regarding the combination of MPN with TFS, reduced gains are found in comparison with the Non-TFS+MPN case.

The additional gain obtained with TFS and MFRP depends on the number of frequency reuse patterns that can be configured for a given reuse factor. For $\eta=4$ and $\eta=7,3$ and 2 different patterns can be found, respectively, whereas for $\eta=3$ there is only one. The most important gains come from 
TABLE II

NSE (BPS/Hz) WITH PURE MFNS FOR $\eta=3, \eta=4$ AND $\eta=7$. CASE $D_{T X}=60 \mathrm{KM}, h_{e} f=250 \mathrm{M}$. FULLY CORRELATION (C) AND UNCORRELATED VARIANTS U1 AND U2.

\begin{tabular}{cccccccccc} 
& \multicolumn{3}{c}{$\eta=3$} & \multicolumn{3}{c}{$\eta=4$} & \multicolumn{3}{c}{$\eta=7$} \\
\hline \hline Network Configuration & C & U1 & U2 & C & U1 & U2 & C & U1 & U2 \\
\hline Non-TFS & 1.27 & 1.35 & 1.33 & 1.12 & 1.20 & 1.16 & 0.98 & 1.00 & 0.97 \\
\hline Non-TFS + MPN & 1.31 & 1.39 & 1.34 & 1.24 & 1.30 & 1.24 & 0.99 & 1.02 & 0.98 \\
\hline TFS & 1.63 & 1.77 & 2.36 & 1.40 & 1.50 & 2.06 & 1.13 & 1.19 & 1.54 \\
\hline TFS + MPN & 1.68 & 1.79 & 2.42 & 1.50 & 1.60 & 2.14 & 1.16 & 1.20 & 1.56 \\
\hline TFS + MFRP & - & - & - & 1.49 & 1.96 & 2.11 & 1.18 & 1.39 & 1.57 \\
\hline TFS + MPN + MFRP & - & - & - & 1.59 & 2.05 & 2.20 & 1.20 & 1.43 & 1.60 \\
\hline
\end{tabular}

the MFRP+MPN case when considering the time uncorrelated approach since there exist large differences in the received signal level from different stations at each given point in the time domain. As said before, the network with $\eta=3$ cannot benefit from the gains provided by MFRP. With $\eta=7$ the reduced number of multiple patterns reduces the achievable gain for this frequency reuse, in contrast to $\eta=4$.

The most relevant gains are achieved with the combination of TFS, MPN and MFRP (up to 89\% gain with $\eta=4$ uncorrelated U2 and $42 \%$ with the fully-correlated approach). The use of TFS+MFRP and TFS+MPN+MFRP provides higher NSE for $\eta=4$ than the maximum achievable with $\eta=3$ (with TFS+MPN) for the uncorrelated approach U1. Therefore, the random variability of the signals coming from different stations in a real network may lead to high gains. Note that when considering the GE06 plan MFN reference network with $\eta=7$, TFS and ANP already provide gains, reaching a minimum gain of $22 \%$ NSE increase when combining all the techniques. In general, the most important gains with the fully uncorrelated (U2) approach are found for TFS whereas the effects of including MFRP or MPN are limited.

Although in this section only results for the $D_{T X}=60$ $\mathrm{km}, h_{\text {eff }}=250 \mathrm{~m}$ configuration are displayed by means of figures, it is worth to comment that the NSE increases for the $D_{T X}=80 \mathrm{~km}, h_{\text {eff }}=400 \mathrm{~m}$. In general, for the same transmitter distance, NSE increases with effective transmitter antenna height. NSE increase is, however, reduced (around $10 \%$ gain for the TFS+MPN+MFRP uncorrelated case).

For comparison purposes, MFN with $\eta=1$ has also been calculated. It provides the lowest NSE (around $0.5 \mathrm{bps} / \mathrm{Hz}$ for the Non-TFS case and around $1 \mathrm{bps} / \mathrm{Hz}$ with TFS+MPN. In this case the wanted hexagon always present an adjacent CCI cell.

2) MFNs of SFN Clusters and Large area SFNs: Table III compiles the NSE reached within MFNs made of SFN clusters. The cluster size is 7 transmitters, all of them using the same polarization. Results are presented for frequency reuse factors 3 and 4 within the $D_{T X}=60 \mathrm{~km}$ and $h_{\text {eff }}=250 \mathrm{~m}$ scenario. Since it is not possible to implement more than one frequency reuse pattern with $\eta=3$, the entries of the table involving this situation are not filled.

Results show that the network with frequency reuse $\eta=3$ is more spectral efficient than when $\eta=4$ is implemented. In the first case the main differences arise when using TFS with respect to the reference Non-TFS case due to its impact
TABLE III

NSE (BPS/Hz) WITH MFNS OF SFN CLUSTERS FOR $\eta=3$ AND $\eta=4$. CASE $D_{T X}=60 \mathrm{KM}, h_{e f f}=250 \mathrm{M}$. FULLY CORRELATION (C) AND UNCORRELATED VARIANTS U1 AND U2.

\begin{tabular}{ccccccc} 
& \multicolumn{3}{c}{$\eta=3$} & \multicolumn{3}{c}{$\eta=4$} \\
\hline \hline Network Configuration & C & U1 & U2 & C & U1 & U2 \\
\hline Non-TFS & 2.29 & 2.40 & 2.28 & 1.82 & 1.85 & 2.17 \\
\hline Non-TFS + MPN & 2.30 & 2.41 & 2.29 & 1.87 & 1.90 & 2.21 \\
\hline TFS & 2.58 & 2.76 & 3.36 & 2.03 & 2.13 & 3.29 \\
\hline TFS + MPN & 2.59 & 2.77 & 3.38 & 2.11 & 2.12 & 3.30 \\
\hline TFS + MFRP & - & - & - & 2.20 & 2.68 & 3.48 \\
\hline TFS + MPN + MFRP & - & - & - & 2.29 & 2.72 & 3.54 \\
\hline
\end{tabular}

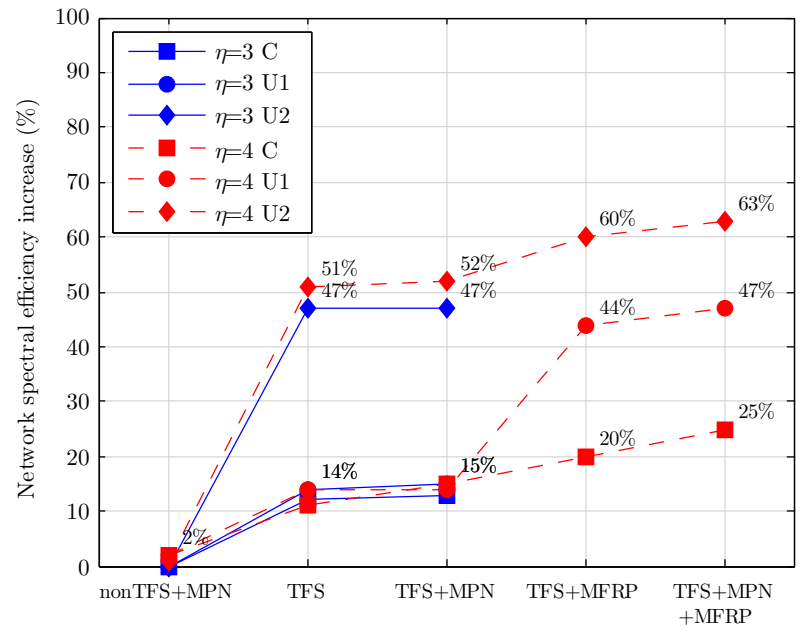

Fig. 11. NSE increase $(\% \mathrm{bps} / \mathrm{Hz})$ for MFNs of SFN clusters with reuse factor 3 and 4. For each reuse factor and network type, the Non-TFS case is taken as reference. Transmitter distance $D_{T X}=60 \mathrm{~km}$ and effective antenna height $h_{\text {eff }}=250 \mathrm{~m}$. Fully correlation (C) and uncorrelated variants U1 and U2.

on averaging imbalances between RF channels. Fig. 11 also presents the NSE increase achieved by each configuration.

With $\eta=3$ and 4 the performance of MPN is low since it is not possible to mitigate the interferences coming from the 7 transmitters in the most interfering clusters. The limited effect of MPN and the non-existence of more than a single frequency reuse pattern for $\eta=3$ do not provide important additional gains to TFS.

High NSE is achieved with $\eta=4$ for MFRP and the 
TABLE IV

NSE (BPS/Hz) WITH LARGE AREA SFNS FOR $\eta=3$ AND $\eta=4$. CASE $D_{T X}=60 \mathrm{KM}, h_{e f f}=250 \mathrm{M}$. FULLY CORRELATION (C) AND UNCORRELATED VARIANTS U1 AND U2.

\begin{tabular}{ccccccc} 
& \multicolumn{3}{c}{$\eta=3$} & \multicolumn{3}{c}{$\eta=4$} \\
\hline \hline Network Configuration & C & U1 & U2 & C & U1 & U2 \\
\hline Non-TFS & 1.94 & 1.99 & 1.94 & 1.46 & 1.50 & 1.77 \\
\hline Non-TFS + MPN & 2.18 & 2.21 & 2.15 & 1.65 & 1.64 & 2.00 \\
\hline TFS & 2.23 & 2.33 & 2.98 & 1.68 & 1.74 & 2.79 \\
\hline TFS + MPN & 2.48 & 2.54 & 3.24 & 1.86 & 1.90 & 3.04 \\
\hline TFS + MFRP & - & - & - & - & - & - \\
\hline TFS + MPN + MFRP & - & - & - & - & - & - \\
\hline
\end{tabular}

combination of MFRP and MPN configurations. The most relevant gains are found for TFS in combination with ANP. In particular, the gain for TFS+MPN+MFRP reaches $20 \%, 44 \%$ and $60 \%$ with $\eta=4$, for the fully-correlated and uncorrelated approaches $\mathrm{U} 1$ and $\mathrm{U} 2$, respectively.

Regarding the configuration $80 \mathrm{~km}$ transmitter distance and $400 \mathrm{~m}$ effective antenna height, which results are not graphically presented, although the NSE is larger, its increase with respect to the Non-TFS case is reduced (around $60 \%$ to $50 \%$ for the TFS+MPN+MFRP case).

Again, $\eta=1$ provides the lowest NSE for the different cases under analysis. The results are similar as in a MFN due to the existence of adjacent co-channel stations to the edge of the SFN.

NSE has been calculated for the large area SFN case as shown in Table IV. It may be possible to implement MFRPs in a large area SFN network with the frequency reuse factors $\eta=3$ and $\eta=4$ used in this study. However, this case is not taken into account since the most important interference contributions come from the same SFN due to self-interference when the delay between the signals coming from two transmitters in the SFN exceed the permitted delay to be considered as constructive interferences.

Large area SFN networks can benefit from the effects of using MPN to mitigate the self-interferences. Likewise, the use of TFS alone or in combination with MPN provides a $C /(N+I)$ increase. Fig. 12 shows the gains achieved for large area SFNs and ANP.

If compared to the network of SFN clusters the use of TFS and TFS with MPN provides higher advantage in the large area SFN network. This is mainly due to the fact that potential CCI stations are located closer to the receiver location than those CCI from a co-channel cluster. Conversely, the decreased $\mathrm{C} /(\mathrm{I}+\mathrm{N})$ in a large area SFN provides lower NSE.

\section{B. Application of the results}

The three different network topologies (MFN, MFNs of SFN Clusters and Large area SFNs) are compared with respect to the GE06 reference network with $\eta=7$ and Non-TFS. The analysis is made considering frequency reuse factors $\eta=4$ which can exploit all the proposed ANP strategies. The achievable gains are computed considering the results derived from the 3 correlation approaches. Table $\mathrm{V}$ collects the maximum and minimum NSE increase derived from simulations.

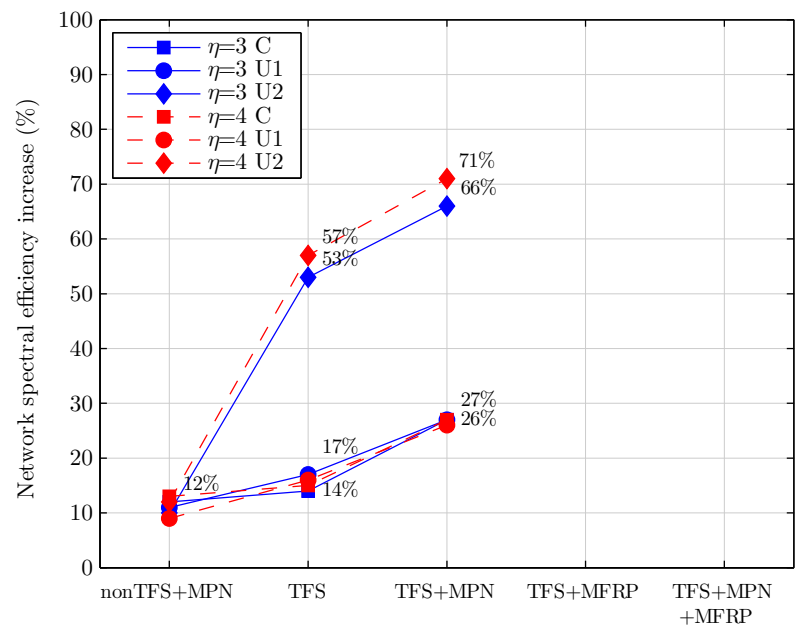

Fig. 12. NSE increase $(\% \mathrm{bps} / \mathrm{Hz})$ for Large SFNs with reuse factor 3 and 4. For each reuse factor and network type, the Non-TFS case is taken as reference. Transmitter distance $D_{T X}=60 \mathrm{~km}$ and effective antenna height $h_{\text {eff }}=250 \mathrm{~m}$. Fully correlation (C) and uncorrelated variants U1 and U2.

TABLE V

MINIMUM AND MAXIMUM NSE INCREASE (\%) FOR FREQUENCY REUSE FACTOR $\eta=4$ COMPARED TO THE NON-TFS MFN $\eta=7$ CASE (GE06 PLAN).

\begin{tabular}{ccccccc} 
& \multicolumn{2}{c}{ MFN } & \multicolumn{2}{c}{ SFN Clusters } & \multicolumn{2}{c}{ Large-SFN } \\
\hline \hline Network Configuration & Min & Max & Min & Max & Min & Max \\
\hline Non-TFS & 14 & 19 & 85 & 123 & 48 & 82 \\
\hline Non-TFS + MPN & 26 & 30 & 89 & 127 & 63 & 106 \\
\hline TFS & 42 & 112 & 107 & 239 & 71 & 187 \\
\hline TFS + MPN & 53 & 120 & 112 & 240 & 89 & 213 \\
\hline TFS + MFRP & 52 & 117 & 124 & 258 & - & - \\
\hline TFS + MPN + MFRP & 62 & 126 & 133 & 264 & - & - \\
\hline
\end{tabular}

It can be seen that all of the topologies analysed provide higher NSE than the reference $\eta=7$ network, even without TFS. MFNs provide the lowest increase. However, the maximum increase for the MFN with $\eta=4$ reach more than $100 \%$ when using TFS and TFS with ANP. Large area SFN networks do not provide as much NSE increase as the MFNs made of SFN clusters. The use of MFNs of SFN clusters outperform any other network topology. The MFN with SFN clusters and $\eta=4$ can obtain a high performance when combining TFS and ANP. In general, the NSE within the network of SFN clusters is higher than for a pure MFN since the co-channel stations inside each cluster do not create interferences, and the cluster size allows for increased reuse distances for a given reuse factor. Furthermore, with SFN clusters, the 7 stations in each interfering cluster reach the reception point in the SFN with similar field strength (similar distances) what makes $C /(N+I)$ decrease.

NSE gains with ANP are also evaluated considering limited transmitter power (ERP). In general terms, NSE values decrease whereas NSE gain is increased when decreasing ERP. Similar values as those presented in this paper apply for a large range of ERP from 10 to $100 \mathrm{~kW}$, which are typical ERP used by transmitter DTT stations. It should be 
noted that the gains reached by the use of TFS or TFS with MFRP collapse to the gains reached by only TFS with low ERP and MFNs. The combination of TFS with MPN achieves higher gain that the only TFS case also with low ERP and MFNs. For SFN clusters, the combination of TFS with MPN or MFRP collapses to the gain of only TFS for low ERP. Thus, in a real network where ERP is limited to a certain (high) value, TFS and ANP could provide higher NSE gains when using tighter frequency reuse than those achieved in the pure interference-limited cases. The reduction of interference levels in the MFN has a more important effect than the achievable reduction in SFNs.

DTT networks designed for wide area coverage using fixed roof-top reception also allows for portable/mobile reception within a limited area, in which coverage is typically noise limited. The portable/mobile reception therefore normally has some interference margin. When TFS/ANP is applied, and introduced together with a reduction in frequency reuse factor, the general C/I level will increase, but the fixed roof-top coverage would be maintained thanks to TFS/ANP, as shown in this study, but with a larger total capacity (more multiplexes will fit the available spectrum thanks to the lower frequency reuse factor). The portable/mobile reception would also experience an increase in general interference level, for which TFS/ANP would provide some protection, although probably less than for fixed reception, due to lack of antenna azimuth discrimination and lower XPD performance. If the net effect is negative the original interference margin would be reduced or even be negative, depending on the use case. Indoor reception, having a higher path loss, would have a higher interference margin and is therefore less likely to experience a negative effect on coverage.

Finally, from the point of view of TFS and ANP, the number of RF channels has an impact on the achievable performance. It is important to note that in order to exploit the benefits of the MFRP approach there should be at least as many RF channels as pattern versions for a given reuse factor. With frequency reuse 4, 3 different MFRPs are found. Thus, optimum performance is found when each frequency of the TFS-Mux can experience a different reuse pattern, what is achievable when there exist 3 or more RF channels in the TFS-Mux. Frequency distribution within the band is also an important consideration since frequencies with a large frequency separation suffer from a higher field strength imbalance. For a case with the same ERP being used on all RF frequencies (traditional DTT situation due to regulation constraints) the reception of the Non-TFS cases is limited by the RF channel with the lowest field strength (in average, the RF channel with the highest frequency). Higher TFS gains are expected when there is a high RF channel within the TFS-Mux. Results are also obtained for 3 and 2 RF channels within the TFS-Mux. The selected frequencies are $514 \mathrm{MHz}-554 \mathrm{MHz}-594 \mathrm{MHz}$ and $514 \mathrm{MHz}$ - $594 \mathrm{MHz}$, respectively. The reuse patterns that provide the highest performance are selected. For frequency reuse factor $\eta=4$ and for the fully correlation case (C) the NSE increase with 3 and 2 RF channels is $17 \%$ and $30 \%$ for MFNs; $19 \%$ and $14 \%$ for MFNs of SFN clusters; and, $22 \%$ and $19 \%$ for Large area SFNs.
Note also that the advantages of TFS and TFS with ANP will not be exploited by those RF channels that cannot be aggregated into a TFS-Mux. Conversely, those RF channels could still benefit from the use of MPNs.

\section{CONCLUSIONS}

The results presented in this study reflect the potential benefits of implementing TFS and ANP strategies over a DTT network in a regular hexagonal lattice. Thus, the related gains are not conclusive and will differ for different network topologies and real deployment scenarios.

The increased interference immunity offered by TFS may be exploited in a tighter frequency reuse together with an appropriately chosen capacity/robustness combination, which maximizes the overall capacity per transmitter station of the total available spectrum. Furthermore, network planning could be optimized taking the characteristics of TFS into account. Different ANP strategies allow for higher network spectral efficiency per RF channel by means of different reuse patterns for the frequencies in use (MFRP) and the use of systematic $\mathrm{H} / \mathrm{V}$ polarization at different sites (MPN).

The increased robustness against interferences by TFS and ANP can lead to the same capacity per multiplex being achieved with $\eta=3$ or $\eta=4$ instead of $\eta=7$. This would directly imply a network spectral efficiency gain of $75 \%$ without increasing the bit rate per multiplex (retaining the same coverage). In the possible situation of a $700 \mathrm{MHz}$ band release a total of $28 \mathrm{UHF}$ channels will remain for terrestrial broadcasting. $\eta=7$ will only made 4 RF channels per station available. A new plan with $\eta=4$ will increase the number of multiplexes per station from 4 to 7 , thus achieving the same total capacity as the current DTT network.

The introduction of ANP techniques can increase network spectral efficiency not only for pure MFNs but also for SFN, especially for networks using SFN clusters which size is dimensioned according to GI and transmission mode. The achievable gains provided by TFS in combination with ANP for $\eta=4$ are found to range $41 \%-89 \%, 25 \%-63 \%$ and $26 \%-71 \%$ for MFN, MFN of SFN clusters and large SFN deployments, respectively. Although the reality may not be $100 \%$ de-correlation or $100 \%$ correlation, these results suggest that TFS combined with advanced network planning may offer very high network spectral efficiency gains.

Note that the obtained gain figures apply within the context of simulations using a theoretical regular network deployment and standard terrain characteristics. The achievable gains in a real network deployments can be higher or lower according to the particularities of the network and the level of interferences reaching the receiver. High interference levels on particular RF channels will cause a severe impact on the performance of the Non-TFS case since it will limit the reception of the complete service offering. Conversely, high gains of TFS and TFS with ANP are expected in such cases. Achievable gains are also sensitive to the number of RF channels in the TFS-Mux and the interference levels suffered in each frequency.

As future work, the study of the ANP topologies over non-regular networks and real deployments is proposed in 
order to evaluate the performance of TFS and TFS with ANP with more realistic assumptions regarding the shape, size of the networks with their heterogeneous characteristics and propagation characteristics.

\section{REFERENCES}

[1] "Trends in Broadcasting: An Overview of Developments," ITU, Tech. Rep., Feb 2013.

[2] J. Zander and P. Mahonen, "Riding the data tsunami in the cloud: myths and challenges in future wireless access," Communications Magazine, IEEE, vol. 51, no. 3, pp. 145-151, March 2013.

[3] "Spectrum limit masks for digital terrestrial television broadcasting," ITU, Recommendation ITU-R BT.1206-1, 2013.

[4] I. Eizmendi, M. Velez, D. Gomez-Barquero, J. Morgade, V. BaenaLecuyer, M. Slimani, and J. Zoellner, "Dvb-t2: The second generation of terrestrial digital video broadcasting system," Broadcasting, IEEE Transactions on, vol. 60, no. 2, pp. 258-271, June 2014.

[5] "Digital dividend: Insights for spectrum decisions," ITU, Tech. Rep., Aug 2012.

[6] W. Zhang, Y. Wu, N. Hur, T. Ikeda, and P. Xia, "FOBTV: Worldwide Efforts in Developing Next-Generation Broadcasting System," Broadcasting, IEEE Transactions on, vol. 60, no. 2, pp. 154-159, JUNE 2014.

[7] T. K. Tan, M. Mrak, V. Baroncini, and N. Ramzan, "HEVC Verification Test Results," MPEG, Tech. Rep. JCTVC-Q0204, 2014.

[8] T. O'Leary, E. Puigrefagut, and W. Sami, "GE06 - overview of the second session (RRC 06) and the main features for broadcasters," no. 308, Oct 2006.

[9] J. J. Giménez, E. Stare, S. Bergsmark, and D. Gómez-Barquero, "Time frequency slicing for future digital terrestrial broadcasting networks," Broadcasting, IEEE Transactions on, vol. 60, no. 2, pp. 227-238, June 2014.

[10] Frame structure channel coding and modulation for a second generation digital terrestrial television broadcasting system (DVB-T2), ETSI Std. EN 302 755, Rev. V1.3.1, April 2012.

[11] D. Gómez-Barquero, C. Douillard, P. Moss, and V. Mignone, "DVBNGH: the Next Generation of Digital Broadcast Services to Handheld Devices," Broadcasting, IEEE Transactions on, vol. 60, no. 2, pp. 246257, 2014.

[12] C. Shannon, "A mathematical theory of communication," Bell system technical journal, vol. 27, p. 379423 and 623656, 1948.

[13] J. Zander, S.-L. Kim, M. Almgren, and O. Queseth, Radio Resource Management for Wireless Networks. Norwood, MA, USA: Artech House, Inc., 2001.

[14] "Directivity and polarization discrimination of antennas in the reception of television broadcasting," ITU, Recommendation ITU-R BT.419-3.

[15] R. Vaughan, "Polarization diversity in mobile communications," Vehicular Technology, IEEE Transactions on, vol. 39, no. 3, pp. 177-186, Aug 1990.

[16] M. Makni, J. Robert, and E. Stare, "Performance analysis of time frequency slicing," in Electronic Media Technology (CEMT), 14th ITG Conference on, 2011, pp. 1-6.

[17] D. Gozalvez, J. Gimenez, D. Gomez-Barquero, and N. Cardona, "Rotated constellations for improved time and frequency diversity in dvbngh,” Broadcasting, IEEE Transactions on, vol. 59, no. 2, pp. 298-305, June 2013

[18] "Frequency and network planning aspects of DVB-T2," European Broadcasting Union, Tech. Rep. 3384, Nov 2013.

[19] R. Brugger and D. Hemingway, "OFDM Receivers. Impact on Coverage of Inter-Symbol Interference and FFT Window Positioning," EBU, Tech. Rep. Jul, 2003.

[20] "Report from ad-hoc group B/CAI-FM24 to B/MDT \& FM PT24 on spectrum requirements for DVB-T implementation," European Broadcasting Union, Tech. Rep. 023, Oct 2013.

[21] "Method for point-to-area predictions for terrestrial services in the frequency range $30 \mathrm{MHz}$ to $3000 \mathrm{MHz}$," ITU, Recommendation ITU-R P.1546-5, 2013.

[22] S. R. Saunders and A. Aragón Zavala, Antennas and Propagation for Wireless Communication Systems, 2nd ed. New York, NY, USA: John Wiley \& Sons, Inc., 2007.

[23] R. Fraile, J. F. Monserrat, J. Gozálvez, and N. Cardona, "Mobile radio bi-dimensional large-scale fading modelling with site-to-site crosscorrelation," European Transactions on Telecommunications, vol. 19, no. 1 , pp. 101-106, 2008.
[24] J. J. Giménez, D. Gozálvez, D. Gómez-Barquero, and N. Cardona, "Statistical model of signal strength imbalance between RF channels in DTT network," Electronics Letters, vol. 48, no. 12, pp. 731-732, 2012.

[25] "Propagation effects relating to terrestrial land mobile and broadcasting services in the VHF and UHF bands," ITU, Recommendation ITU-R P.1406-1, 2007.

[26] D. Plets, W. Joseph, P. Angueira, J. Arenas, L. Verloock, and L. Martens, "On the methodology for calculating sfn gain in digital broadcast systems," Broadcasting, IEEE Transactions on, vol. 56, no. 3, pp. 331339, Sept 2010.

[27] C. Tunc, A. Altintas, and V. Erturk, "Examination of existent propagation models over large inhomogeneous terrain profiles using fast integral equation solution," Antennas and Propagation, IEEE Transactions on, vol. 53, no. 9, pp. 3080-3083, Sept 2005.

[28] M. Waddell, "TVWS Opportunities. Evaluating Coexistence Parameters," in 3rd IEEE BTS GOLD Workshop. Broadcasting in the IP Era, May 2014.

[29] "Radiation pattern characteristics of UHF television receiving antennas," ITU, Tech. Rep. ITU-R BT.2138.

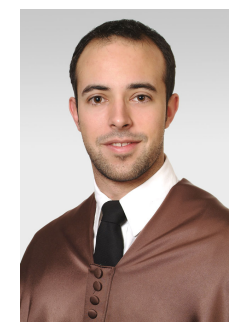

Jordi Joan Giménez received the M.Sc. degree in Telecommunications Engineering from the Universitat Politècnica de València (UPV), Spain, in 2010. He joined Institute of Telecommunications and Multimedia Applications (iTEAM) of UPV, in 2010, where he carried out the M.Sc. Thesis, which was awarded by the Official College of Telecommunication Engineers of Spain. He is currently a $\mathrm{PhD}$ student with UPV supported by a PhD scholarchip for reseach training from UPV. He has participated in the Celtic-Plus ENGINES project, in the standardization process of DVB-NGH inside the Framing, Sounding and Synchronization working group and in the DVB MIMO study mission. During 2013 and 2014, he was a Guest Researcher at Teracom AB and Wirelesss@KTH in Sweden, respectively. His $\mathrm{PhD}$ thesis topic and research interests include the characterization and modeling of radio propagation and advanced network planning techniques for next-generation DTT networks.

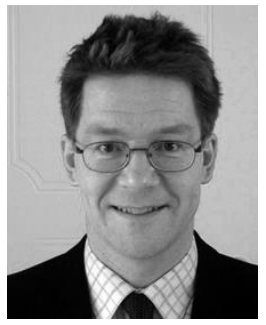

Erik Stare received a Master of Science in Electrical Engineering in 1984 from the Royal Institute of Technology (KTH) in Stockholm. He joined Ericsson in 1984 and from 1987 Swedish Telecom (now TeliaSonera) where he was involved in R\&D projects related to HDTV and digital terrestrial TV. Since 1992 he has worked at Teracom, mainly with $R \& D$ related to various aspects of digital terrestrial TV/HDTV. He has been deeply involved in the development and standardisation of the broadcast systems DVB-T, DVB-H, DVB-T2/T2-Lite and DVBNGH and is the inventor of MPE-FEC in DVB-H and Time Frequency Slicing in DVB-T2 and DVB-NGH. He has participated in a number of European $\mathrm{R} \& \mathrm{D}$ projects under the ACTS, IST and CELTIC programmes. 


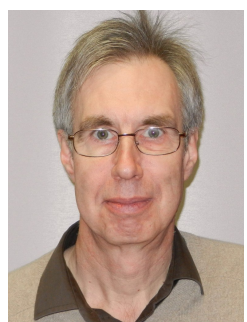

Staffan Bergsmark was born in Stockholm, Sweden, in 1953. He received the Master of Science degree in Electrical Engineering from the Royal Institute of Technology (KTH), Stockholm, Sweden in 1981. In 1981, he joined Swedish Telecom (now TeliaSonera) where he worked with satellite telecommunication. Since 1992, he has been with Teracom. His area of work includes development of digital COFDM TV system, planning parameters for digital TV, verification of SFN networks, frequency planning of digital TV networks, mobile diversity reception of DVB-T, field measurement of system performs, coverage measurement of broadcast systems, network planning for TFS. He has participated in European R\&D projects under the ACTS, IST and CELTIC programmes. $\mathrm{He}$ is involved in the standardization of planning parameters in CEPT and ITU.

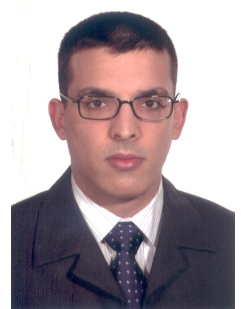

David Gómez-Barquero received the double M.Sc. degree in telecommunications engineering from the Universitat Politècnica de València (UPV), Spain, and the University of Gävle, Sweden, in 2004, and the Ph.D. degree in telecommunications from UPV in 2009. During his doctoral studies, he was a Guest Researcher at the Royal Institute of Technology, Sweden, the University of Turku, Finland, and the Technical University of Braunschweig, Germany. He also did an internship at Ericsson Eurolab, Germany. From 2010 to 2011, he was a Post-Doctoral Guest Researcher at the Fraunhofer Heinrich Hertz Institute, Germany. He is currently a Senior Researcher (Ramon y Cajal Fellow) at the Institute of Telecommunications and Multimedia Applications (iTEAM), UPV, where he leads a research group working on multimedia broadcasting, in particular on the optimization of 3GPP multimedia broadcast multicast services (MBMS) and digital video broadcasting (DVB) systems. Since 2008, he has been actively participating in the European digital television standardization forum DVB. He participated in the validation of DVB-T2, and in the standardization processes of its mobile profile T2-Lite and its handheld evolution DVB-NGH He also contributed to the DVB-T2 implementation guidelines, and co-edited the implementation guidelines for upper layer forward error correction. $\mathrm{He}$ was very involved in the promotion and adoption of DVB-T2 in Colombia, and since the second half of 2012 he has been working with the spectrum regulator on the spectrum and network planning and optimization of DVB-T2. Since then, he is also a Visiting Professor at the Sergio Arboleda University of Bogota, Colombia. Dr. Gómez-Barquero is the Editor of the book, Next Generation Mobile Broadcasting (CRC Press), and the Vice-Chairman of the Modulation and Coding Ad-Hoc Group of the ATSC 3.0 standardization process. 